\title{
A Percepção de uma Comunidade Quilombola a Respeito de Leishmaniose Visceral, em Mato Grosso do Sul e a Educação em Saúde como Ferramenta de Controle Desta Endemia
}

\section{The Perception of a Quilombola Community Regarding Visceral Leishmaniosis, in Mato Grosso do Sul and Health Education as a Tool to Control This Endemic}

\author{
Suellem Luzia Costa Borges*a; Gilberto Gonçalves Faccoa; Rafael Ovando Fraiha ${ }^{\mathrm{b}}$; João Cledson Pedrozo Rigo ; \\ Dina Regis Recaldes Rodrigues Argeropulos Aquino ${ }^{\text {d }}$ Eduardo de Castro Ferreira ${ }^{\text {ab }}$
}

\begin{abstract}
${ }^{a}$ Universidade Anhanguera Uniderp, Programa de Pós-Graduação Stricto Sensu Meio Ambiente e Desenvolvimento Regional. MS, Brasil. bUniversidade Federal de Mato Grosso do Sul, Programa de Pós-Graduação Stricto Sensu em Doenças Infecciosas e Parasitárias. MS, Brasil. 'Instituto de Pós-Graduação Qualittas. MS, Brasil.

dUniversidade Anhanguera Uniderp, Curso de Medicina Veterinária. MS, Brasil.

*E-mail: suellemlcb@gmail.com
\end{abstract}

\begin{abstract}
Resumo
As leishmanioses são zoonoses de grande distribuição global, que apresentam várias espécies de reservatórios e vetores, que ao se relacionarem no tempo e espaço estabelecem os ciclos de transmissão dessa doença negligenciada, cujas estratégias de controle devem ser estruturadas sob a perspectiva da Saúde Única, que tem como premissa a interconectividade da saúde humana, animal e ambiental. O objetivo do estudo foi avaliar a percepção da população residente em uma Comunidade Quilombola, no interior de Mato Grosso do Sul, no que se refere às leishmanioses, assim como discutir a importância da educação em saúde como estratégia de prevenção. Estudo seccional, analítico, transversal, em uma Comunidade Quilombola, pertencente ao município de Jaraguari, Mato Grosso do Sul, com setenta indivíduos selecionados por conveniência e de acordo com os critérios de elegibilidade, representando suas famílias. A Comunidade estudada aparenta ter pouco conhecimento sobre leishmaniose e estratégias de educação e saúde focadas ao tema, baseadas em uma perspectiva multidisciplinar, conforme preconizado pelos defensores da saúde única devem ser pautadas pelas equipes de saúde do município a que pertence. Os dados revelam eminência de uma epidemia canina, pois o ambiente é favorável e a densidade vetorial é abundante, o que normalmente antecede à proliferação de casos humanos. Palavras-chave: Meio Ambiente. Educação em Saúde. Zoonoses. Saúde Única
\end{abstract}

\begin{abstract}
Leishmaniasis are zoonoses of great global distribution, which present several species of reservoirs and vectors, which, when relating in time and space, establish the transmission cycles of this neglected disease, whose control strategies should be structured under the perspective of Saúde Única, which has as assumption the interconnectivity of human, animal and environmental health. The objective of this study was to evaluate the population perception residing in a Quilombola community, in the interior of Mato Grosso do Sul state, regarding leishmaniasis, as well as to discuss the importance of health education as a prevention strategy. Sectional, cross-sectional, analytical study in a Quilombola community, belonging to the municipality of Jaraguari, Mato Grosso do Sul, with seventy individuals selected for convenience and according to the eligibility criteria, representing their families. The studied Community seems to have little knowledge about leishmaniasis and education and health strategies focused on the theme, based on a multidisciplinary perspective, as recommended by the advocates of Saúde Unica should be guided by the health teams of the municipality to which it belongs. Data reveal the eminence of a canine epidemic, since the environment is favorable and vector density is abundant, which usually precedes the human cases proliferation.
\end{abstract}

Keywords: Environment. Education in Health. Zoonoses. Saúde Única

\section{Introdução}

As leishmanioses são zoonoses que apresentam distribuição global e compõem o grupo de doenças negligenciadas, ocorrendo principalmente em países com pouco acesso aos serviços de saúde e parcela significativa da população em condição de vulnerabilidade, como aqueles que se localizam na África, Ásia e Américas.

A presença de várias espécies de mamíferos, que possam atuar como reservatórios, assim como as diversas espécies de insetos vetores, que ao se relacionarem no tempo e espaço estabelecem os ciclos de transmissão, indicam que o controle dessas doenças somente terá sucesso se as estratégias adotadas forem realizadas no âmbito da Saúde Única, o qual reconhece que a saúde humana está diretamente ligada à saúde animal e ambiental, sendo este um elemento fundamental para monitorar e controlar as ameaças à saúde pública (CDC, 2013).

Em relação à leishmaniose visceral (LV), cerca de 96\% dos casos registrados nas Américas foram notificados no Brasil, entretanto, países Sul-Americanos como Argentina, Colômbia, Paraguai e Venezuela têm registrado um incremento constante no número de casos (OPAS, 2019).

No Brasil, a doença é causada pelo protozoário Leishmania (Leishmania) infantum e transmitida pela picada de insetos vetores - Lutzomyia longipalpis e Lutzomyia cruzi - infectados, conhecidos popularmente como mosquito palha, tatuquiras, birigui, entre outros. Além do homem, que representa um hospedeiro acidental da doença, ao entrar em contato com o ciclo de transmissão de seu agente etiológico, esta parasitose acomete várias outras espécies de mamíferos, animais como roedores, marsupiais, edentados, quirópteros e canídeos, entre 
outros (CARREIRA, 2012; CASTRO et al., 2020).

É uma doença grave com poucas opções terapêuticas e que, mesmo quando adequadamente tratada, tem letalidade de cerca de 5\% (WERNECK, 2010).

Em virtude do significativo aumento da incidência (28\%), quando comparados os anos de 2016 e 2017, observa-se interesse crescente nesta parasitose no Brasil (OPAS, 2019). Além de relato de epidemias recentes, em áreas endêmicas, há evidências de disseminação para áreas anteriormente não endêmicas. Tais situações podem ser explicadas, em parte, pela melhoria do diagnóstico e notificação dos casos, mas também são resultados de fatores associados ao aumento da movimentação da população, migrando da área rural para urbana, do surgimento de resistência a drogas utilizadas no tratamento e à adaptação dos ciclos de transmissão aos ambientes peridomiciliares, decorrentes da urbanização e do desmatamento (GONTIJO; MELO, 2004; FRANÇA-SILVA et al., 2005; OLIVEIRA et al., 2006; BRASIL, 2014)

Neste cenário epidemiológico, é consenso que o conhecimento da população em relação à incidência, às formas de transmissão e à prevenção traz contribuições relevantes para o controle da enfermidade. Além disso, a investigação do comportamento e das crendices da população pode também orientar as práticas de prevenção, tornando-as mais efetivas (LUZ, SCHALL e RABELO, 2005; BORGES et al., 2008; MARGONARI et al., 2012).

Dessa forma, o objetivo do presente estudo foi avaliar a percepção da população residente em uma Comunidade Quilombola, no interior de Mato Grosso do Sul, no que se refere às leishmanioses, assim como discutir a importância da educação em saúde como estratégia de prevenção.

\section{Material e Métodos}

Foi realizado um estudo seccional, analítico, transversal, em uma Comunidade Quilombola, pertencente ao município de Jaraguari, Mato Grosso do Sul, sem registros de casos humanos, embora apresente condições ambientais favoráveis ao estabelecimento do ciclo de transmissão.

Esta comunidade é composta por 89 famílias, porém, de acordo com o cálculo amostral, na qual a amostragem foi delineada a partir de Fonseca e Martins (1994) dever-seia totalizar 72 famílias pesquisadas, no entanto, conforme os critérios de elegibilidade, duas famílias não aceitaram participar, ou não se encontravam na residência em nenhuma das datas de visita.

A seleção das amostras ocorreu por conveniência, respeitando os aspectos éticos (Chamada FUNDECT/DECITMS/CNPq/SES N 04/2013 - PPSUS-MS, Termo de Outorga 0056/13), procedimento este autorizado pelo Comitê de Ética em Pesquisa da Universidade Anhanguera Uniderp, parecer 2.325.203 (CAAE: 75362117.7.0000.5161), com recrutamento nos dias 25/08, 20/10/2018, 23/03/2019 e 18/09/2019, conforme a disponibilidade oferecida pela comunidade.
Foram entrevistadas 70 famílias, representadas por um membro eleito para responder às questões, por meio de visita domiciliar, agendado previamente com o agente comunitário e o presidente da referida Comunidade.

A coleta de dados ocorreu por meio de entrevista individual, utilizando o questionário do tipo semiestruturado, seguindo o modelo adaptado de Gama et al. (1998) e Borashi (2007), sendo aplicado pelo próprio pesquisador, no período supracitado, destacando variáveis demográficas, socioeconômicas e de conhecimento.

Como critérios de elegibilidade são considerados maiores de 18 anos, na residência no momento da visita, morador da Comunidade, concordar com a pesquisa e assinar o Termo de Consentimento Livre e Esclarecido.

Comentários estatísticos: a análise se fundamentou na Estatística Descritiva, com ênfase nos valores relativos.

\section{Resultados e Discussão}

Foram pesquisados 70 indivíduos Quilombolas, residentes na Comunidade de Furnas do Dionísio, município de Jaraguari, MS. A população se enquadra com Índice de Massa Corporal (IMC) médio normal e com as referidas comorbidades presentes: $21(30 \%)$ relatam Hipertensão Arterial Sistêmica (HAS), 06 (8,5\%) com Diabetes mellitus (DM), 03 (4,2\%) com anemia e destes apenas um não está em tratamento (hipertenso).

O Quadro 1 expressa o perfil sócio demográfico da população pesquisada, apresentando predominância do sexo feminino, jovem, com Ensino Fundamental completo, renda entre um e dois salários mínimos.

Quadro 1 - Perfil demográfico da população pesquisada em Furnas do Dionísio, Jaraguari - MS ( $\mathrm{n}=70)$

\begin{tabular}{|l|c|c|}
\hline Perfil Demográfico & N & \% \\
\hline Sexo & & \\
\hline Feminino & 47 & 67,14 \\
\hline Masculino & 23 & 32,85 \\
\hline Faixa Etária & & \\
\hline $18-28$ anos & 20 & 28,57 \\
\hline $29-39$ anos & 14 & 20,00 \\
\hline $40-50$ anos & 14 & 20,00 \\
\hline $51-61$ anos & 14 & 20,00 \\
\hline$>61$ anos & 08 & 11,42 \\
\hline Renda Familiar & & \\
\hline$<1$ Salário Mínimo (SM) & 26 & 37,14 \\
\hline$>1$ SM $<2$ SM & 38 & 54,28 \\
\hline$>2$ SM $<3$ SM & -- & --- \\
\hline$>3$ SM $<4$ SM & -- & --- \\
\hline$>4$ SM & -- & --- \\
\hline Não Informou & 06 & 8,57 \\
\hline Escolaridade & & \\
\hline Analfabeto & 02 & 2,85 \\
\hline Ensino Fundamental Completo & 22 & 31,42 \\
\hline Ensino Fundamental Incompleto & 15 & 21,42 \\
\hline Ensino Médio Completo & 07 & 10,00 \\
\hline Ensino Médio Incompleto & 16 & 22,85 \\
\hline Superior Completo & 08 & 11,42 \\
\hline Fonte: Dados da pesquisa & & \\
\hline
\end{tabular}

Fonte: Dados da pesquisa. 
Quanto à ocupação, 48,57\% relatam serem lavradores, $32,85 \%$ dona de casa, $1,42 \%$ aposentado, $2,85 \%$ da construção civil, 2,85\% gerais, 2,85\% autônomo, $4,28 \%$ desempregado e $4,28 \%$ não informaram.

Segundo dados do IBGE (2010), o município de Jaraguari - MS se caracteriza em predomínio de população jovem, com mudança da pirâmide etária nos últimos anos. Em 2017, a renda média mensal era de 2,1 salários mínimos. Apresenta 2,7\% de domicílios com esgotamento sanitário adequado, 95\% de domicílios urbanos em vias públicas com arborização e $10,1 \%$ destes com presença de esgotamento sanitário e pavimentação asfáltica (IBGE, 2017).

Galastro e Fonseca (2006) entendem que o determinismo social imposto pelo sistema capitalista brasileiro faz transparecer a figura feminina ligada à subalternidade dos processos produtivos e secundário em relação ao masculino, principalmente, ligada aos filhos, no qual a mulher assume o papel do cuidado e dos afazeres domésticos, independentemente de exercer sua força produtiva remunerada.

Borges et al. (2008), Martins et al. (2018) e Sousa et al. (2018) constataram que a variável escolaridade demonstrou que quanto menos se frequenta a escola, maior a chance de ser acometido por leishmaniose visceral. Essa associação pode ser explicada pela maioria dos analfabetos estarem entre a população de baixo poder aquisitivo, em que a situação de vulnerabilidade social, que também é a que mais sofre com a doença, segundo Bevilacqua et al. (2001).

Parte da população em estudo possui água encanada, $34,28 \%$ relatam desfrutar desta condição e os demais utilizam água de poço; quanto ao esgoto tratado, 100\% responderem não possuir, mencionando o uso de fossa (10\%) ou de despejo a céu aberto. A comunidade é desprovida de asfaltamento e não há coleta de lixo sistematizada, dessa forma, os indivíduos o acondicionam em lata tampada $(8,57 \%)$ ou lata sem tampa $(11,42 \%)$ ou em sacos plásticos $(21,42 \%)$ ou colocam em um buraco e queimam de tempos em tempos, sem uma definição exata de data $(60 \%)$.

Dos entrevistados, 74,28\% residem em casa de alvenaria, $25,71 \%$ consideraram que sua casa é apenas rebocada, sem pintura e $100 \%$ descreveram que o telhado é apenas com telha, sem forro. O piso de $47,14 \%$ é de cimento, $44,28 \%$ de cerâmica e 8,6\% referem madeira ou terra batida.

Em relação aos aspectos de condições sanitárias, estão diretamente relacionadas aos criadouros, manutenção e proliferação de vetores da leishmaniose, principalmente, ao que se refere à coleta de lixo e tratamento de esgoto. Em Furnas do Dionísio, em relação à coleta de lixo se constatou que não há recolhimento sistematizado e que $60 \%$ desta comunidade tem o hábito de queimá-lo de tempos em tempos. Em relação à rede de esgoto do município de Jaraguari, sua abrangência é extremamente baixa quando comparada à média da região Centro - Oeste $(50,4 \%)$ e a Comunidade é $100 \%$ desprovida, isso ocorre, principalmente, pela localização em zona rural.

A exemplo deste estudo, Gama et al. (1998), no Maranhão, constataram que a área pesquisada (local de grande número de casos de LV), localizava-se em região periurbana do município, observando-se precariedade no saneamento básico e esta variável também está presente em diversos outros levantamentos (CESSE, 1999; CAVALCANTI et al., 2017).

Compreendendo que o espaço e a condição de vida representam mediações passíveis de informar relações entre a sociedade e sua saúde, correlacionando maiores incidências de leishmaniose visceral humana e piores condições de vida, como no estudo de Toledo et al. (2017).

Diversos estudos mostram que as características de moradia influenciam diretamente a transmissão das leishmanioses, afirmando que esta doença é mais incidente em áreas de baixo nível socioeconômico, com casas de taipa, cobertas de palha e piso de terra batida (ALENCAR; FIGUEIREDO, 2018; BORASCHI; PERRI; NUNES, 2008; CAVALCANTI et al., 2017; CESSE, 1999; GAMA et al., 1998; MESTRE; FONTES, 2007), pois os espaços nas paredes possibilitam a circulação do vetor e/ou servem como abrigos, assim como as casas sem forração podem permitir a entrada de insetos vetores, principalmente, durante à noite, conforme o observado em parte da população estudada, caracterizada como de alvenaria $(74,28 \%)$ e sem forro $(100 \%)$.

Importante ainda relatar que $87,14 \%$ dos quintais são de terra batida e embrenhados à mata (Quadro 2), sendo 94,28\% com algum tipo de criação de animais (como, por exemplo, galinhas em 75,71\% das residências), além disso, a maioria possui árvore frutífera $(52,21 \%)$ e/ou horta (20,35\%) (Quadro 2).

Quadro 2 - Características do quintal e ações de prevenção da LV pela população pesquisada em Furnas do Dionísio, Jaraguari - MS

\begin{tabular}{|l|c|c|}
\hline \multicolumn{1}{|c|}{ Piso do Quintal } & N & \% \\
\hline Cimentado & 04 & 5,71 \\
\hline Cerâmica & --- & --- \\
\hline Gramado & 05 & 7,14 \\
\hline Terra Batida & 61 & 87,14 \\
\hline Características Gerais* & & \\
\hline Árvores frutíferas & 59 & 52,21 \\
\hline Horta & 23 & 20,35 \\
\hline Umidade & 08 & 7,07 \\
\hline Material orgânico em decomposição & 08 & 7,07 \\
\hline Lixo & 09 & 7,96 \\
\hline Entulhos & --- & --- \\
\hline Mortes recentes de animais & 06 & 5,30 \\
\hline Ação de Prevenção no Quintal & & \\
\hline Quintal sempre limpo* & 65 & 46,42 \\
\hline Recolher fezes dos animais, diariamente* & 12 & 8,57 \\
\hline $\begin{array}{l}\text { Recolher folhas e frutos do chão, } \\
\text { diariamente* }\end{array}$ & 18 & 12,85 \\
\hline Lixo sempre tampado* & 10 & 7,14 \\
\hline $\begin{array}{l}\text { Evitar adentrar à mata fechada sem } \\
\text { proteção* }\end{array}$ & 08 & 5,71 \\
\hline Usar repelente* & 11 & 7,85 \\
\hline Janelas teladas* & 08 & 5,71 \\
\hline Usar mosqueteiros* & 5,71 \\
\hline
\end{tabular}




\begin{tabular}{|l|c|c|}
\hline $\begin{array}{l}\text { Presença de Telas é uma Medida de } \\
\text { Prevenção? }\end{array}$ & & \\
\hline Sim & 02 & 2,85 \\
\hline Não & 68 & 97,14 \\
\hline Utiliza Tela em sua Residência? & & \\
\hline Sim, nas janelas & & --- \\
\hline Sim, nas janelas e canil & & --- \\
\hline Não & 70 & 100,00 \\
\hline
\end{tabular}

*cabia mais de uma resposta.

Fonte: Dados de pesquisa.

As características do quintal, ou peridomicílio, e as ações praticadas pelos indivíduos pesquisados, que podem contribuir com a prevenção da transmissão de LV ou a diminuição do risco de LV, naquela região estão presentes no Quadro 2.

Observa-se que tais condições são favoráveis à formação de criadouros de flebotomíneos, o que, em conjunto ao déficit das ações de prevenção à $L V$, podem contribuir para o surgimento de novos casos na região.

Compreender o processo de organização do espaço geográfico pelas sociedades humanas, em diferentes tempos e lugares, é uma forma particular de entender as doenças, pois expressam valores funcionais e simbólicos (LEMOS; LIMA, 2002; CASTRO et al., 2016). Porém, apenas essa variável isolada não se faz essencial à transmissão. A deficiência no saneamento básico, baixos índices socioeconômicos, a convivência com animais domésticos, resultando em acúmulo de matéria orgânica, o desmatamento e a invasão de nichos ecológicos proporcionam condições favoráveis para a ocorrência da transmissão de doenças (BRASIL, 2006; MONTEIRO et al., 2005; TAUIL, 2006), além de possíveis mudanças na ecologia e biologia de vetores (COSTA, 2008).

No peridomicílio de $21,42 \%$ dos indivíduos pesquisados há a presença de terreno baldio; 94,28\% apresentam mata, reflorestamento e criação de animais em geral, principalmente, galinhas $(75,71 \%)$, porco $(34,28 \%)$, vaca/cavalos $(2,85 \%)$, pássaros $(2,85 \%)$ e pato $(4,28 \%)$; o cão está presente em $88,57 \%$ das residências, sendo que $8,06 \%$ dos moradores relataram que o animal está doente. Dos entrevistados, 41,42\% mencionaram que já viu algum animal silvestre perto de sua residência, destacando que $58,62 \%$ citam ratos.

A presença de animais domésticos atrai a aproximação e permanência do inseto vetor no peridomicílio, em função da possibilidade de servirem como fonte de alimento, uma vez que os flebotomíneos fêmeas são hematófagas.

Diversos estudos discursam a respeito do ciclo enzoótico de transmissão da Leishmania infantum (possíveis variáveis) no ecótopo peridoméstico, constatando que a proximidade da mata, a altitude das residências e a visita de gambás ao peridomicílio podem ser assumidas como fatores de risco para as leishmanioses, assim como a pluviosidade, o terreno plano, a presença de florestas, de bovinos e a fruticultura (CABRERA, 1999; CABRAL, 2007; MARTINS et al., 2004; MARGONARI et al., 2012; MONTEIRO et al., 2005; SILVA et al., 2010).
Os galinheiros e as pocilgas também tiveram grande participação nos resultados da pesquisa de Forattini, Rabelo e Galati (1976); Souza et al. (1981); Galati et al. (1995); Azevedo et al. (2000); Alexander et al. (2002); Moreno et al. (2005); Oliveira et al. (2006); Reinhold-Castro et al. (2008); Boraschi, Perri e Nunes, (2008) e Nunes et al. (2008); Dorval et al. (2009).

As profundas transformações ambientais antrópicas favorecem a adaptação e a formação de novos criadouros de flebotomíneos, agravadas por fatores socioeconômicos representadas em condições precárias de habitação, moradias irregulares e de infraestrutura sanitária, além de baixos níveis nutricionais (ALVES et al., 2018; BRASIL, 2010; CAMARGO-NEVES; GOMES; ANTUNES, 2002; MARZOCHI; MARZOCHI, 1997; MESTRE; FONTES, 2007; MISSAWA; LIMA, 2006).

Uma abordagem como essa se enquadra no conceito de Saúde Única, que tem como possíveis benefícios maior possibilidade de prevenção e combate às doenças parasitárias, mas a prática da Saúde Única de maneira eficaz se faz necessária a sensibilização dos gestores de saúde, que devem estar cientes da importância da participação de profissionais de diferentes áreas no processo de tomada de decisão e na área da saúde básica (CIRNE; CABRERA, 2019). Segundo Day (2010), médicos, veterinários, outros profissionais de saúde, pesquisadores de órgãos governamentais e não governamentais devem trabalhar juntos para garantir a preservação da saúde em suas mais diversas formas.

A ocorrência da doença em uma determinada área depende, basicamente, da presença do vetor e de um hospedeiro/ reservatório suscetíveis, o que em frequência (número absoluto) os mais encontrados na região de estudo, em trabalho paralelo realizado por nosso grupo, foram Lutzomyia longipalpis seguida de Nyssomyia whitmani e Evandromyia lenti (dados não publicados), assim como observado por Bastos, Linhares e Madrid (2016) em região urbana de Goiás, em que $90 \%$ foi capturado em canil e $9 \%$ em galinheiros.

Os hospedeiros silvestres de $L$. infantum até agora conhecidos são as raposas e os marsupiais, o que demonstra mais uma variável de risco eminente, pois $41,42 \%$ dos entrevistados já visualizaram animais silvestres próximos a sua residência e $88,57 \%$ \% possuem o cão como animal doméstico.

No ambiente doméstico, o cão é considerado um importante hospedeiro e fonte de infecção para os vetores, sendo um dos alvos nas estratégias de controle. Entretanto, para se determinar o papel destes animais na manutenção da transmissão ainda são necessários maiores estudos (GONTIJO; MELO, 2004).

Vale ressaltar que o teste rápido para leishmaniose visceral canina, $D P P \AA$, realizado por este grupo de pesquisa foi positivo em amostras de 10/24 cães (41,66\%), entre esses, oito animais apresentavam sinais clínicos compatíveis com LVC, sendo mais frequentes as lesões de pele, onicogrifose, secreção ocular bilateral e linfadenomegalia. A positividade entre os cães ainda está em avaliação por meio de outras 
técnicas de diagnóstico, mas já indicam uma possibilidade eminente de epidemia de leishmaniose visceral humana na comunidade, o que influencia o indicador de periculosidade da comunidade.

Sabe-se que a epidemia canina normalmente precede o aparecimento dos casos humanos, como ocorreu no Nordeste brasileiro e em Belo Horizonte (DEANE, 1956; BEVILACQUA et al., 2001, OLIVEIRA; ARAUJO, 2003) e que tem relação direta com a presença no peridomicílio de animais domésticos e sinantrópicos (DIAS; LOROSA; REBELO, 2003).

Considerando o conhecimento geral que a população entrevistada tem em relação à LV (Quadro 3) se observa que apesar de 97,14\% dos indivíduos mencionarem que já ouviram falar a respeito deste agravo, $86,76 \%$ não conhecem outro nome para a doença e $7,35 \%$ a confundiram com barriga de água (esquistossomose).

Quadro 3 - Conhecimento sobre leishmaniose visceral da população pesquisada em Furnas do Dionísio, Jaraguari - MS $(\mathrm{n}=70)$

\begin{tabular}{|c|c|c|}
\hline Conhecimento Geral & $\mathbf{N}$ & $\%$ \\
\hline \multicolumn{3}{|l|}{ Já Ouviu Falar Sobre LV? } \\
\hline Sim & 68 & 97,14 \\
\hline Não & 02 & 2,86 \\
\hline \multicolumn{3}{|l|}{ Onde Ouviu Falar? } \\
\hline ACS* & 18 & 26,47 \\
\hline Amigos & 15 & 22,05 \\
\hline Escola & 11 & 16,17 \\
\hline TV & 14 & 20,58 \\
\hline Não sabe informar & 03 & 4,41 \\
\hline Outros** & 07 & 10,29 \\
\hline \multicolumn{3}{|l|}{ Outros Nomes Para a LV? } \\
\hline Barriga de água & 05 & 7,35 \\
\hline Doença do cão & 03 & 4,41 \\
\hline Ferida Brava & 01 & 1,47 \\
\hline Não sabe & 59 & 86,76 \\
\hline \multicolumn{3}{|l|}{ Forma De Transmissão da LV? } \\
\hline Mordida do cão & 10 & 14,28 \\
\hline Mosquito Aedes aegypti & 12 & 17,14 \\
\hline Urina do rato & 01 & 1,42 \\
\hline Vento & 01 & 1,42 \\
\hline Não sabe & 46 & 65,71 \\
\hline \multicolumn{3}{|c|}{ Animal que Também Apresenta LV? } \\
\hline Cachorro & 19 & 27,14 \\
\hline Gato & 02 & 2,85 \\
\hline Não sabe & 49 & 70,0 \\
\hline \multicolumn{3}{|l|}{ Quem Transmite? } \\
\hline Cão doente & 20 & 28,57 \\
\hline Mosquito & 12 & 17,14 \\
\hline Gato & 02 & 2,85 \\
\hline Besouro & 01 & 1,42 \\
\hline Não sabe & 36 & 51,42 \\
\hline \multicolumn{3}{|l|}{ Outro Nome do Transmissor? } \\
\hline Aedes aegypti & 09 & 12,85 \\
\hline Borrachudo & 05 & 7,14 \\
\hline Não Sabe & 56 & 80,00 \\
\hline
\end{tabular}

*Agente Comunitário de Saúde. ${ }^{* * H}$ Hospital, os pais, palestras e equipe de vacinação de cães.

Fonte: Dados da pesquisa.
Quando a população pesquisada foi indagada a respeito da fonte de informação sobre a LV, destaca-se a influência das mídias de comunicação e do ACS neste processo. Quanto ao conhecimento sobre a transmissão da LV, observou-se que $17,14 \%$ consideram que ocorre por meio do Aedes aegypti ou não sabem responder $(65,71 \%)$. Quanto ao nome do transmissor, nenhum indivíduo respondeu corretamente e $80,0 \%$ relataram não saber (Quadro 3). Em relação a conhecer o período de maior ocorrência da doença, 12,85\% citam o período de chuva, $8,57 \%$ de seca e $78,57 \%$ não sabem responder.

Ao serem questionados a respeito de que se algum familiar já teve LV, 98,57\% relataram que desconhecem casos positivos e o mesmo padrão de resposta apresentado quando perguntados acerca de casos positivos na proximidade.

Em relação se algum animal seu já desenvolveu lesão/ ferida, $17,14 \%$ relataram que sim; destes, 75,0\% mencionam o cão (lesões múltiplas e há mais de quatro anos) e 16,66\% em bovino.

Apenas 1,42\% (01/70) dos moradores utiliza como medida preventiva citronela tópica e plantou citronela no quintal; porém, destaca-se que $98,57 \%$ não utilizam nenhuma medida. $\mathrm{O}$ participante que executa as medidas refere que foi orientado pelo veterinário.

Sobre o desejo de aprender sobre a LV, 97,14\% gostariam de conhecer mais. Nesta comunidade, $86,76 \%$ dos entrevistados não sabem quais nomes o vetor da leishmaniose pode apresentar, já que os vetores do agente etiológico da LV são insetos que possuem nomes diferenciados, de acordo com a região, os mais conhecidos são flebótomo, mosquito palha, tatuquiras e birigui.

Segundo Borges et al. (2008), em Belo Horizonte, pessoas que dizem conhecer, ao menos um nome dado ao vetor, têm 1,58 vezes mais chance de serem acometidas pela leishmaniose visceral que aquelas que desconhecem os nomes, enquanto que em Mossoró (RN) (COSTA et al., 2014) esta probabilidade se estendeu ao dobro de risco. Essa associação pode ser explicada pelo fato desse conhecimento existir em função de um contato prévio com a doença, seja por pessoas ou animais próximos infectados.

Diante dessas variáveis, ressaltar a importância do conhecimento das medidas protetivas é essencial para minimizar o risco, porém não o suficiente, deve haver coerência entre conhecimentos e atitudes. Para que isso ocorra se deve considerar os aspectos culturais que envolvem o cotidiano do indivíduo (BORGES et al., 2008; CAMARGO; BARBARÁ, 2004; FAVARO; QUEIROZ; NUNES, 2017).

Esta Comunidade demonstrou conhecimento baixo em relação à leishmaniose visceral e as medidas protetivas, pois apesar de $97,14 \%$ já terem ouvido falar sobre a doença, $65,71 \%$ desconhecem a forma e o período de transmissão $(78,57 \%)$ e $70 \%$ desconhecem qual animal pode apresentar a doença. 
Sabe-se que o conhecimento se constrói no sujeito, que é tarefa de significação (apropriação), e para que isto ocorra é necessário a vinculação da informação aos contextos e experiências do indivíduo. Este processo é lento, reflexivo e individual, ainda que o produto do conhecimento seja, a posteriori, socializado (BARRETO, 2006), em relação às doenças, esta falta de conhecimento leva ao retardo na procura do diagnóstico e do tratamento, sendo as populações rurais de áreas endêmicas as mais carentes de informação (GAMA et al., 1998).

E nesta região estudada, a presença do Agente Comunitário de Saúde se destacou em relação àqueles que já ouviram falar, assim como a televisão, porém não há a fomentação desta informação transmitida, característica também presente no estudo realizado por Anversa, Montanholi e Sabino (2016), em Bauru, Lopes et al. (2019) no Maranhão e Youssef et al. (2019) em Marília.

Em relação às atribuições dos $\mathrm{ACS}$, de acordo com a Portaria GM/MS 2436/2017, destaca-se estar em contato permanente com as famílias, desenvolvendo ações educativas, visando promoção da saúde e prevenção das doenças, de acordo com o planejamento da equipe; além da vigilância à saúde, por meio de visitas domiciliares e de ações educativas individuais e coletivas nos domicílios e na comunidade, mantendo a equipe informada, principalmente, a respeito daquelas em situação de risco, reforçando Uchoa et al. (2004), os quais compreende que a disseminação das informações é de grande valia para o estabelecimento de campanhas de controle.

Moura e Moretti (2003) afirmam que o processo de aprendizagem envolve aspectos globais como a disposição dos ouvintes, os instrumentos, as habilidades e o conhecimento prévio que os indivíduos possuem acerca do assunto, assim como afetividade, disposição, interesse, ambiente social, interações e a relação do conceito com a realidade concreta.

Dessa forma, uma aprendizagem será significativa quando o receptor for capaz de estabelecer relações coerentes entre o que já sabe e o novo conhecimento que lhe está sendo apresentado. Para tanto, Piaget afirma que "a inteligência humana somente se desenvolve no indivíduo em função de interações sociais que são, em geral, negligenciadas".

Santos et al. (2019) afirmam que as medidas de proteção são fatores importantes, pois se a maioria da população ignora estes aspectos, consequentemente, poderá não ocorrer a adesão, a adequação e a correção em níveis permanentes e satisfatórios ao uso destas, o que leva a uma falha do programa de controle e prevenção à LV. Assim, torna-se imperativo a adesão e aceitação da população em relação a essas medidas. Na comunidade, a população refere que não acredita que a colocação de telas nas janelas seja uma medida protetiva $(97,14 \%)$, mas sim a limpeza do quintal $(46,42 \%)$ e o recolhimento de flores e frutos que caem no chão (12,85\%).

Borges et al. (2008) estimam que haja diminuição de 1,94 vezes no risco de ocorrência de leishmaniose visceral para pessoas que declaram tomar qualquer atitude preventiva, quando comparadas às que não praticam tais ações.

A pouca informação sobre o processo de transmissão da LV, traduzida nesses resultados, constitui uma preocupação, tendo em vista que quando não se conhece a respeito da doença, a adesão às medidas de prevenção/proteção, tornase lento e mínimo. Por isso, destaca-se aqui a importância da discussão a respeito da educação em saúde.

Tal fato aponta para a necessidade da realização de práticas educativas em diferentes frentes, que possam contar com a participação de profissionais de saúde durante as consultas, professores e agentes de saúde em mobilizações constantes e durante as visitas domiciliares (ALENCAR; FIGUEIREDO, 2018; BORGES et al., 2008). Demonstra, também, a necessidade peculiar de esclarecimentos quanto às nomenclaturas básicas, a fim de que a informação possa permear mais claramente o intelecto e sensibilizar a população ouvinte.

Luz, Schall e Rabello (2005) citam a educação como controle cultural para as leishmanioses, por tornar participantes diversas camadas da população e por democratizar atitudes capazes de beneficiar as práticas de controle.

Nas áreas de transmissão intensa, bem como nas áreas cobertas pelas Equipes de Agentes Comunitários de Saúde (EACS) e Equipe de Saúde da Família (ESF), é recomendada a busca ativa de casos e o encaminhamento dos suspeitos para atendimento médico. Dessa forma, é importante que os profissionais estejam sensibilizados para a problemática e a população informada sobre a patologia, além da necessidade de buscar atendimento precocemente, conhecer a disponibilidade dos serviços de saúde oferecidos (BRASIL, 2006a) e que haja incremento de ações ambientais em Saúde Pública e amadurecimento das ações sob a ótica do desenvolvimento sustentável (VARRICCHIO et al., 2019) e da Saúde Única (CDC, 2013).

A dificuldade apontada por esta comunidade se direciona em relação ao acesso ao serviço de saúde, pois a unidade de saúde local está desativada e o vínculo é estreitado pelo ACS, que encaminha as necessidades à unidade urbana.

Porém, para que haja melhor entendimento dos riscos de transmissão e da vulnerabilidade, a qual cada indivíduo está sujeito, faz-se necessário que as equipes de atendimento da área urbana ampliem sua capacidade diagnóstica e terapêutica, na rede de saúde desta comunidade, e priorizem as áreas de intervenção e de ênfase na educação em saúde.

Pimenta, Leandro e Schall (2007) relatam que a maioria dos materiais educativos ainda utiliza a imagem em movimento como mera ilustração e descrição do "real", sendo as leishmanioses e seu portador meros "objetos" de descrição e ilustração. Ao mesmo tempo em que se simplificam certas informações, até em excesso, em alguns casos, infantilizando e menosprezando o espectador, sendo utilizados jargões técnicos ou classificações científicas, para um público especializado da área biomédica, dificultando o entendimento 
da população.

Por isso, a equipe de profissionais da saúde deve conhecer seu público e tornar a informação mais palatável, voltado às crenças, à cultura e aos hábitos comuns àqueles que desfrutam deste processo.

É importante ressaltar que a linguagem tem que estar a favor da cidadania, deve ser uma reinvenção dessa, que é uma das questões centrais da educação popular, uma vez que não importa apenas o conteúdo a ser abordado, mas também a forma com que isto acontece, tendo como grande objetivo a liberdade, e não apenas repasse de comunicados (FREIRE, 2001).

Porém, para que o indivíduo se sinta parte deste processo se faz necessário profissionais capacitados e disponíveis a educar, permeando suas inquietações e desvendando as dúvidas a serem esclarecidas, nesse aspecto, Santos et al. (2019) destacam o profissional enfermeiro. Nesse sentido, o saber profissional está na confluência entre várias fontes de saberes provenientes da história de vida individual, da sociedade, da instituição escolar, dos outros atores educativos, dos lugares de formação, além de uma abordagem simplificada (TARDIF; RAYMOND, 2000)

\section{Conclusão}

Conclui-se que a Comunidade estudada tem baixo conhecimento sobre leishmaniose e estratégias de educação e saúde focadas no tema, baseadas em uma perspectiva multidisciplinar, conforme preconizado pelos defensores da saúde única, devem ser pautadas pelas equipes de saúde do município a que pertence. Os dados revelam eminência de uma epidemia de LV baseado em fatores ambientais favoráveis, a alta densidade vetorial e, sobretudo, o registro de casos caninos o que, normalmente, antecede aos casos de leishmaniose humana.

A comunidade se mostra disposta a aprender e a compartilhar saberes, porém os profissionais da saúde precisam dialogar e abrir as fronteiras do conhecimento, explorando o vínculo e formando novos multiplicadores, prezando por uma dimensão educativa, baseada na autonomia individual e coletiva.

A população tem direito a estratégias direcionadas que auxiliam à compreensão mais diretiva dos riscos que estão sujeitos, assim como suas condutas para minimizá-los.

\section{Agradecimentos}

Ao fomento de PPSUS, FUNDECT, UNIDERP e PAPES VII -FIOCRUZ / CNPq.

\section{Referencias}

ALENCAR, B.F.P; FIGUEIREDO, I.A. Perfil epidemiológico dos casos de Leishmaniose Tegumentar Americana no estado do Maranhão no período de 2015 a 2017. Rev. Investig, Bioméd, v.10, n.3, p.243-250, 2018. doi: 10.24863/rib.v10i3.340

ALEXANDER, B. et al. Role of the domestic chicken (gallus gallus) in the epidemiology of urban visceral leishmaniasis in Brasil. Emerg Infect Dis, v.8, n.2, p.1480-1485, 2002.

ANVERSA, L.; MONTANHOLI, R.J.D.; SABINO, D.L. Avaliação do conhecimento da população sobre leishmaniose visceral. Rev Inst Adolfo Lutz, v.75:1685, 2016. doi: https://doi. org/10.1590/S0102-311X1998000200014.

AZEVEDO, A.C.R. et al. Studies on populations of Lutzomyia longipalpis (Lutz \& Neiva, 1912) (Diptera: Psychodidae: Phlebotominae) in Brazil. Mem Inst Oswaldo Cruz, v.95, n.3, p.305-322, 2000.

BARRETO, A.M. Leitura: suas categorias de produção de sentidos nas novas e antigas formas de acesso à informação. Salvador: EDUFBA, 2006.

BASTOS, T.S.A.; LINHARES, G.F.C.L.; MADRID, D.M.C. Identificação morfológica de flebotomíneos capturados em área urbana. Cienc. Anim. Bras, v.17, n.3, p. 395-401, 2016.

BEVILACQUA, P.D. et al. Urbanização da leishmaniose visceral em Belo Horizonte. Arq. Bras. Med. Vet. Zoot., v. 53, n. 1, p. 1-8, 2001.

BORASCHI, C.S.S.; PERRI, S.H.V.; NUNES, C.M. Leishmaniose visceral: o que a população de Três Lagoas, MS, Brasil, sabe sobre esta enfermidade? Vet. Zootec., v.15, n.3, p.478-485, 2008.

BORGES, B.K.A. et al. Avaliação do nível de conhecimento e de atitudes preventivas da população sobre a leishmaniose visceral em belo horizonte, Minas Gerais, Brasil. Cad Saúde Pública, v.24, n.4, p.777-784, 2008.

BRASIL. Ministério da Saúde. Secretaria de Vigilância em Saúde. Departamento de Vigilância Epidemiológica. Doenças Infecciosas e Parasitárias: guia de bolso. Brasília: MS, 2010.

BRASIL. Ministério da Saúde. Manual de vigilância e controle da leishmaniose visceral. Brasília: MS, 2014.

CABRAL, A.P. Influência de fatores ambientais na leishmaniose visceral no Rio Grande do Norte. Natal: Universidade Federal do Rio Grande do Norte, 2007.

CABRERA, M.A.A. Ciclo enzoótico de transmissão da Leishmania (Leishmania) chagasi (Cunha e Chagas, 1937) no ecótopo peridoméstico em Barra de Guaratiba, Rio de Janeiro$R J$ : estudo de possíveis variáveis preditoras. Rio de Janeiro: Fundação Oswaldo Cruz, 1999.

CAMARGO-NEVES, V.L.F.; GOMES, A. C.; ANTUNES, J.L.F. Correlação da presença de espácies de flebotomíneos (Dipteros: Psychodidae) com registros de casos da leishmaniose tegumentar Americana no Estado de São Paulo, Brasil. Rev. Soc. Bras. Med. Tropical, v.35, n.4, p.299-306, 2002.

CAMARGO, B.V.; BARBARÁ, A. Efeitos de panfletos informativos sobre a AIDS em Adolescentes. Psicol. Teoria Pesq., v.20, n.3, p.279-287, 2004.

CARREIRA, J.C. et al. Natural infection of Didelphis aurita (Mammalia: Marsupialia) with Leishmania infantum in brazil. Parasit Vectors, v.5, n.11, p.1-5, 2012.

CAVALCANTI, O.L. et al. Aspectos da incidência de leishmaniose visceral humana e canina no município de Floriano/ PI, Brasil. Rev. Espacios, v. 38, n.8, p. 20-26, 2017.

CDC - Centers for Disease Control and Prevention, National Center for Emerging and Zoonotic Infectious Diseases (NCEZID). One Health Basics, 2018. Disponivel em: <https://www.cdc.gov/ onehealth/basics/index.html> Acesso em: 14 maio 2020.

CESSE, E.A.P. Expansão e urbanização da leishmaniose visceral: estudo epidemiológico do processo de transmissão ativa 
em área urbana - Petrolina/PE, 1992-1997. Recife: Fundação Oswaldo Cruz, 1999.

COSTA, C.H.N.; TAPETY, C.M.M. WERNECK, G.L. Controle da leishmaniose visceral em meio urbano: estudo de intervenção randomizado fatorial. Rev. Soc. Bras. Med. Trop., v.40, n.4, p.415-419, 2007.

COSTA, C.H.N. Characterization and speculations on the urbanization of visceral leishmaniasis in Brazil. Cad. Saúde Pública, v.24, n.12, p.2959-2963, 2008.

COSTA, K.F.L. et al. Awareness of visceral leishmaniasis and its relationship to canine infection in riverside endemic areas of Northeastern Brazil. Rev. Soc. Bras. Med. Trop., v.47, n.5, p.607$612,2014$.

DEANE LM. Leishmaniose visceral no Brasil, estudos sobre reservatórios e transmissores realizados no Estado do Ceará Fortaleza: Serviço Nacional de Educação Sanitária, 1956.

DIAS, F.O.P.; LOROSA, E.S.; REBELO, J.M.M. Fonte Alimentar sanguines e a peridomiciliação de Lutzomyia longipalpis (Lutz \& Neiva, 1912) (Psychodidae, Phlebotominae). Cad. Saúde Pública, v.19, n.5, p.1373-1380, 2003.

DORVAL, ME. et al. Phlebotomine fauna (Diptera: Psychodidae) of an American cutaneous leishmaniasis endemic area in the state of Mato Grosso do Sul, Brazil. Memórias do Instituto Oswaldo Cruz, v.104, n.5, p.695-702, 2009.

FAVARO, A.B.B.B.C.; QUEIROZ, L.H.; NUNES, C.M Conhecimento de diferentes populações sobre as Leishmanioses: uma revisão sistemática. Arch. Health Invest., v.6, n. 6, p.14, 2017.

FORATTINI, O.P.; RABELLO, E.X.; GALATI, E.A.B. Novos encontros de flebotomíneos no Estado de São Paulo, Brasil, com especial referência a Lutzomyia longipalpis. Rev. Saúde Pública, v.10, n.1, p.125-128, 1976.

FRANÇA-SILVA, J.C. et al. Importance of Lutzomyia longipalpis in the dynamics of transmission of canine visceral leishmaniasis in the endemis área of Porteirinha Municipality, Minas Gerais, Brazil. Vet. Parasitol., v.131, n.3-4, p.213-220, 2005.

FREIRE, P. Política e Educação: ensaios. São Paulo: Cortez, 2001.

GALASTRO, E.P.; FONSECA, R.M.G.S. A identidade masculina e feminina na visão dos profissionais de saúde de um serviço de saúde reprodutiva. Rev. Min. Enferm., v.10, n.1, p.37-40, 2006.

GALATI, E.A.B. Phylogenetic systematics of Phlebotominae (Diptera: Psychodidae) with emphasis on American groups. Bol. Diréc. Malariol. Amb., v.35, n.1, p.133-142, 1995.

GAMA, M.E.A. et al. Avaliação do nível de conhecimento que populações residentes em áreas endêmicas têm sobre leishmaniose visceral, Estado do Maranhão, Brasil. Cad. Saúde Pública, v.14, n.2, p.381-390, 1998.

GONTIJO, C.; MELO, M. Leishmaniose visceral no Brasil: quadro atual, desafios e perspectivas. Rev. Bras. Epidemiol., v.7, n.3, p.338-349, 2004.

IBGE - Instituto Brasileiro de Geografia e Estatística. Panorama das Cidades - Censo Demográfico 2010. Disponível em: $<$ https:// cidades.ibge.gov.br/brasil/ms/jaraguari/panorama $>$ Acesso em: 4 abr. 2020 .

LEMOS, J.C.; LIMA, S.C.A geografia médica e as doenças infecto-parasitárias. Caminhos de Geografia, v. 3, n. 6, jun/ 2002.

LOPES, G.S. et al. Nível de conhecimento e medidas de prevenção de moradores sobre a Leishmaniose Visceral em área endêmica no Maranhão, Brasil. Arch. Health Invest., v.8, n.6, p.285-289, 2019.

LUZ, Z.M.P.; SCHALL, V.; RABELLO, A. Evaluation of a pamphlet on visceral leishmaniasis as a tool for providing disease information to healthcare professionals and laypersons. $\mathrm{Cad}$. Saúde Pública, v.21, n.2, p.606-621, 2005.

MANDERSCHEID, J.C. Modèles et príncipes en éducation pour la santé. Rev. Française Pédagogie, v.107, p.81-96,1994.

MARTINS, L.M. et al. Ecoepidemiologia da leishmaniose tegumentar no Município de Buriticupu, Amazônia do Maranhão, Brasil, 1996 a 1998. Cad. Saúde Pública, v.20, n.3, p.735-743, 2004.

MARTINS, C.P.et al. Monitoramento epidemiológico como instrumento de apoio à gestão de saúde: análise das notificações de leishmaniose visceral em Sobral, Ceará. Rev. Adm. Saúde, v.18, n.72, p.1-13, 2018.

MARGONARI, C. et al. Public Knowledge about and Detection of Canine Visceral Leishmaniasis in Urban Divin'opolis, Brazil. J. Trop. Med., 2012. doi: 10.1155/2012/429586.

MARZOCHI, M.C.A.; MARZOCHI, K.B.F. Leishmanioses em áreas urbanas. Rev. Soc. Bras. Med. Trop., v.30, n.1, p.162-164, 1997.

MISSAWA, N.A.; BORBA, J.F. Leishmaniose visceral no município de Várzea Grande, Estado de Mato Grosso, no período de 1998 a 2007. Rev. Soc. Bras. Med. Trop., v.42, n.5, p.496-502, 2009.

MONTEIRO, E.M. et al. Leishmaniose visceral: Estudo de flebotomíneos e infecção canina em Montes Claros, Minas Gerais. Rev. Soc. Bras. Med. Trop.l, v.2, n.38, p.147-152, 2005.

MORENO, E.C. et al. Risk factors for Leishmania chagasi infection in an urban area of Minas Gerais State. Rev. Soc. Bras. Med. Trop., v.38, n.6, p.456-463, 2005.

MOURA, M.O.; MORETTI, V. D. Investigando a aprendizagem do conceito de função a partir dos conhecimentos prévios e das interações sociais. Ciênc. Educ., v.9, n.1, p.67-82, 2003.

NUNES, V.L.B. Ocorrência de leishmaniose visceral canina em assentamento agrícola no Estado de Mato Grosso do Sul, Brasil. Rev. Soc. Bras. Med. Trop., v.3, n.34, p.301-302, 2001.

MESTRE, G.L.C.; FONTES, C.J.F. A expansão da epidemia da leishmaniose visceral no Estado de Mato Grosso, 1998-2005. Rev. Soc. Bras. Med. Trop., v.40, n.1, p.42-48, 2007.

OLIVEIRA, A. L. et al. Foco emergente de leishmaniose visceral em Mato Grosso do Sul. Rev. Soc. Bras. Med. Trop., v.5, n.39, p.446-450, 2006.

OPAS - Organização Panamericana de Saúde. Informe de Leishmanioses, n.7, 2019.

PIMENTA, D.N.; LEANDRO, A.; SCHALL, V.T.A estética do grotesco e a produção audiovisual para a educação em saúde: segregação ou empatia? O caso das leishmanioses no Brasil. Cad. Saúde Pública, v.23, n.5, p.1161-1171, 2007.

RAYMOND, D. Éclatement des savoirs et savoirs en rupture: une réplique à Van der Maren. Rev. Sci. L'éduc.n, v.19, n.1, p.187200, 1993.

REINHOLD-CASTRO, K.R. et al. Avaliação de medidas de controle de flebotomíneos. Rev. Soc. Bras. Med. Trop., v.41, n.3, p.269-276, 2008.

SANTOS, E.S.M. et al. Aspectos Epidemiológicos da Leishmaniose Visceral. REAS/EJCH, 2019. doi: https://doi. org/10.25248/reas.e959.2019

SILVA, A.F.; LATORRE, M.R.D.O.; GALATI, E.A.B. Fatores 
relacionados à ocorrência de leishmaniose tegumentar no Vale do Ribeira. Rev Soc Bras Med Trop., v.43, n.1, p.46-51, 2010.

SOUZA, M. A. et al. Leishmaniose visceral no Rio de Janeiro. 1 Flebotomíneos da área de procedência de caso humano autóctone. Memórias do Instituto Oswaldo Cruz, v.76, n.2. p.161$168,1981$.

SOUSA, N.A. et al. Perfil epidemiológico dos casos de leishmaniose visceral em Sobral-CE de 2011 a 2015. Rev. Senare, v. 17, n.1, p.51-57, 2018.

TARDIF, M.; RAYMOND, D. Saberes, tempo e aprendizagem do trabalho no magistério. Educ. Soc., v.21, n.73, 2000.

TAUIL, P.L. Perspectivas de controle de doenças transmitidas por vetores no Brasil. Rev. Soc. Bras. Med. Trop., v.39, n.3, p.275277, 2006.
VARRICHIO, M.C.B.N. et al. Saúde ambiental: Leishmaniose, produtos naturais, biotecnologia vegetal e desenvolvimento sustentável. Intervozes, v.4, n.1, p,56-68, 2019.

WERNECK, G. L. et al. Avaliação da efetividade das estratégias de controle da leishmaniose visceral na cidade de Teresina, Estado do Piauí, Brasil: resultados do inquérito inicial - 2004. Rev. Epidemiol. Serv. Saúde, v.17, n.2, p.87-96, 2008.

WERNECK, G.L. Expansão geográfica da leishmaniose visceral no Brasil. Cad. Saúde Pública, v.26, n.4, p.644-645, 2010.

YOUSSEF, A.G. et al. Conhecimento populacional sobre leishmaniose no município de Marília, São Paulo, Brasil. Rev. Acad. Ciênc. Anim., v.17, 2019. doi: http://dx.doi. org/10.7213/1981-4178.2019.17008 